\title{
Analysis on Marketing Case of Intel Electronic Products
}

\author{
Niu Jie \\ Tangshan Industrial Vocational Technical College, China \\ niujie43294240@sina.com
}

Keywords: Intel; Electronic products; Marketing

\begin{abstract}
In recent years, macro-economy is rapidly changed with high-speed development of Internet technology. In addition, the change will be further extended in future development, thereby completely changing traditional business model. Mobile Internet produces the most profound influence in many Internet forms, and it is changing development patterns of many industries. However, actual force driving the society to develop to the direction of digitization and informatization is constant innovation of modern information technology and constant reformation of information products. How to realize marketing of electronic products under new situation has become a major topic that should be solved by the electronic industry. In the research of the paper, the author discusses based on the current situation. Electronic product development condition and marketing strategy of Intel Company are combined for in-depth analysis. It is expected that they can play reference value to certain extent.
\end{abstract}

\section{Introduction}

In recent years, information technology revolution has realized leap-forward development under the background of macro-economy high-speed development. Many fields are changed all over the world under such major trend. Theoretical research results and practice data in recent years are combined. It is obvious that the electronic market will enter a stable stage from the perspective of electronic product field. It is difficult to produce more prominent increase then as shown in Table 1.

Table 1 Global consumption electronic market scale

\begin{tabular}{|c|c|}
\hline Year & Total price (billions of dollars) \\
\hline 2010 & 8710 \\
\hline 2011 & 9680 \\
\hline 2012 & 10490 \\
\hline 2013 & 10369 \\
\hline 2014 & 10680 \\
\hline
\end{tabular}

However, electronic products supporting development of Internet and information technology have larger development space. Therefore, it has been widely popular. Current situation shows that China electronic product enterprises still has certain defects in the aspect of marketing. Many enterprises cannot occupy certain space in fierce market competition as shown in Table 2. 
Table 2 Chinese consumption electronic market scale

\begin{tabular}{|c|c|}
\hline Year & Total price (billions of dollars) \\
\hline 2010 & 8442 \\
\hline 2011 & 10215 \\
\hline 2012 & 11400 \\
\hline 2013 & 13910 \\
\hline 2014 & 16325 \\
\hline
\end{tabular}

\section{Marketing Situation and Problems of Electronic Product Market in China}

Chinese electronic product market is developed for short time compared with western countries. It still has large gap from quality inspection in developed countries. Information technology of Chinese electronic industry has actually achieved unprecedented development situation within several decades of years from the perspective of Chinese scope. It experiences the process of nearly 50 years for foreign countries in some aspects. However, it does not mean that electronic products are well developed in China, and it still contains many problems that should be solved. For example, most retail enterprises in China do not have full understanding on E-commerce. Since some electronic enterprise management personnel engage in other traditional industries originally, they do not have sufficient understanding on the emerging electronic market, they still adopt traditional marketing concepts in the links of development and marketing, and different marketing results are obtained. Secondly, since current information development level of retail enterprises is still limited, the development level can be affected by other foreign enterprises in China in some aspects, the distribution and payment are more backward, and it is difficult to combine with market development demand. Finally, the operation and management modes should be further improved due to limitation in the level of consciousness in the aspect of management and operation. It is obvious that there is still a long way for developing electronic products in China, and there are many difficulties and challenges. We should forge ahead and explore boldly.

\section{Analysis on Intel Electronic Products}

Development Process. Intel Company was founded in 1986. Intel Company gradually becomes the largest technology engine for designing and producing semiconductors all over the world currently under the driving role of the market with popularization of PC machines. In Intel Company, main products include processor, chipset, plate and system, software, and other aspects. These products are parts of computer architecture. In recent years, the market share of Intel and its achieved performance are gradually improved, and different strategies are hidden behind the remarkable achievements.

Marketing Channel. Rapid Product Development: Research and development of product at high efficiency in one enterprise is an indispensable important element to enhance the competitiveness of the enterprise in the electronic industry. Product research and development can objectively determine that the product can occupy a place in the market to some extent. It is also related to the product itself and the market competitiveness of the enterprise. Whether a coordinated and adaptive relation can be kept with external market environment at any time or not, and whether product can be fit with main demand in the market are very important for an enterprise especially in the IT industry with rapidly changing market. Intel always insists on such marketing strategy, namely the development speed must be higher than imitation speed.

In fact, Moore-one founder of Intel has put forward the "Moore's Law" as early as in 1965. The law shows that the electronic element quantity contained on the integrated circuit can be increased by one time for every two years, the performance also can be improved by one time, and cost can be reduced 
by one time, such increase can be sustainable, which can be applied to product development of Intel. Intel workers frequently obtained remarkable achievements in the market under the guidance role of the law in subsequent dozens of years, and the speed is too fast that the rival companies are too far behind to catch up with it.

Positive Brand Construction. Intel realized that brand trademark must be firstly established in order to set up own competitive advantage. Therefore, "Intel Inside" was established. It highlights the Intel brand itself on one hand. Meanwhile, it is not just a simple segregation symbol, which also clearly delivers a belief on the other hand, and such belief contains the integration consciousness of the marketing value chain. Firstly, multi-level channel communication is realized through marketing channel. Secondly, recognition of customers on the technology product is strengthened by the aid of channel propaganda. Intel requires that computer manufacturers should specially emphasize 'Intel Inside' in own package and instruction, which not only can highlight Intel brand, but also can stress the importance of the component to computers correspondingly. Intel constantly strengthens the trademark of 'Intel Inside' through various advertisements on one hand, public relations, promotion and various internal and external propaganda activities are also designed specially for it on the other hand. It can be concluded that producers, distributors, consumers, media, financial institutions, shareholders and employees know it very clearly, and all aspects unify understanding, which is also beneficial for forming joint force.

Intel actively establish strong brand in the mind of consumers in the process of consumer brand creation, thereby forming recognition and protection on Intel from the aspect of consumers, and own control force is further strengthened.

High-Quality Marketing Channels. Intel develops relationship with computer manufacturers, channel distributors, etc. as far as possible for long time. Benefit balance of all links in the marketing chain is effectively guaranteed through generous incentives. Good brand relationship is established with stakeholders accordingly. Such incentives are reflected in cooperative advertising and promotion to the whole downstream link.

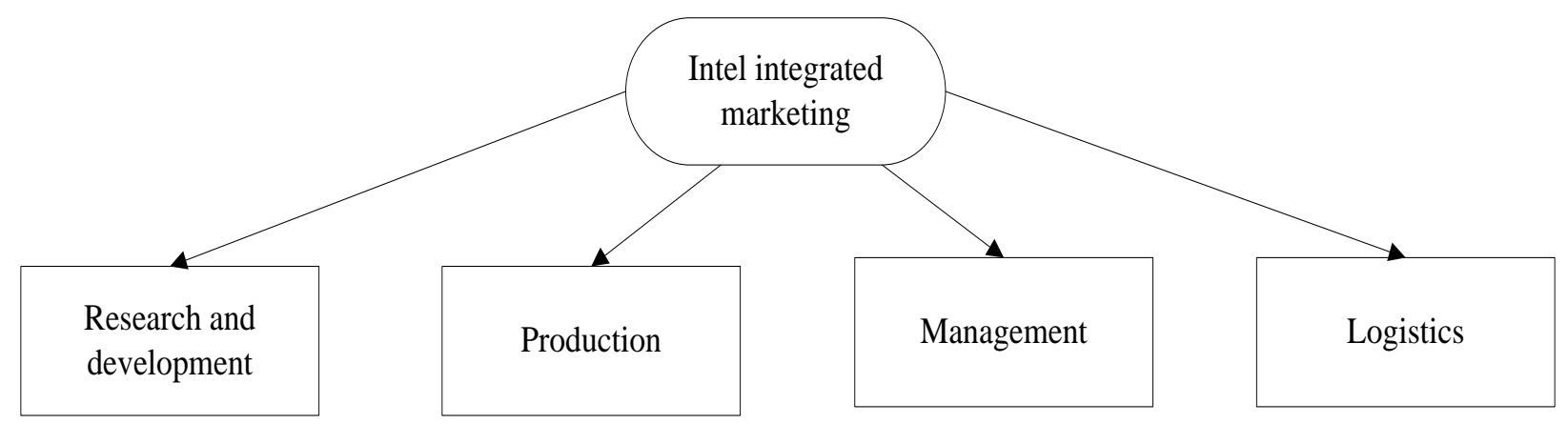

Figure 1. Integrated marketing strategy of Intel

Intel effectively integrates research, production, management and logistics, therefore all links can become strong support factors to 'Intel Inside', and integrated marketing communication can become a brand development strategy related to enterprise operation. When consumers obtain Intel brand information from different communication channels, they firmly believe that Intel is the best micro-processor brand, and can obtain corresponding value satisfaction during purchase. Therefore, Intel also creates consumption value for consumers during creation of brand value.

Demand-Based Pricing Method. Intel Company adopts demand-based pricing method. Concretely, cost is not regarded as basic conditions and main factors during the pricing process. The pricing focuses on understanding and demand of consumers on product value. Then, Intel draws up a price which can be accepted by consumers, and it can occupy certain space in the market. Then, Intel Company will lower the original commodity price according to sales trend and direction as well as similar products produced by competitor, and therefore the market leader can occupy a strong position in competition. Therefore, Intel Company can be far ahead of other competitors in the aspects of 
market share, sales amount, product and technology. The price is higher and higher compared with price of similar products, and Intel price is slightly higher than common price in the market, which can form certain gap from followers in the market.

\section{Enlightenment of Intel Electronic Product Marketing to China}

The most important competition advantages come from two aspects for Chinese enterprises in current complicated and changing international market, one is cost advantage, and the other is product advantage. Chinese enterprises are lack of brand advantages. Therefore, how to establish and improve high-quality brand image of electronic products in China, and rapidly change understanding and recognition of consumers on local products have become urgent.

Establishment of Brand Strategy Consciousness. Previous case analysis shows that brand strategy route has crucial influence on Intel development. Electronic enterprises in China should firstly establish the consciousness of brand strategy, and realize the importance of brand internationalization. In fact, brand internationalization is not a pure economic behavior, and it is also a cultural behavior. Chinese enterprises should make foreign consumers to recognize and comprehend our brand through brand internationalization. It is also an innovation and reforming process, which can effectively cultivate customer loyalty. Brand also can become an important weapon for one enterprise to improve market share and competitiveness under many circumstances as intangible asset in the enterprise operation and management process.

Acquisition of International Influence. First of all, enterprises need to make clear own purpose of realizing internationalization by themselves, some enterprises reduce cost by scale, some enterprises limit impact and influence of competitors through competition in competitors' target market. Electronic enterprises in China are more inclined to the latter. Then, the enterprises should be strictly limited through international standards. Products are sold in the national market objectively, and the same rules must be followed, namely so-called international standard. Chinese enterprises should adopt standard internal control criteria, and control the market by high-quality products, therefore positive competition can be realized. Secondly, market can be created through different demands. Successful of one brand is determined by the following important factor, namely whether the consumption behaviors of consumers in different regions at one time section can be met or not. Therefore, diversified demand in the market should be met for brand marketing. Otherwise, the market cannot be occupied even if the enterprise has advanced technology.

Clear Market Positioning. Enterprise market positioning can be supported in the aspect of issues about market positioning through in-depth market research. So-called marketing positioning refers that enterprises establish distinct enterprise characteristics through own products. Therefore unique impression of clients on products produced by the enterprise can be formed, namely market image. Meanwhile, enterprise should select unique image of products in the enterprise through market during marketing positioning. It is determined that product is placed in a specific position for marketing.

Optimization of Marketing Channel. In Intel's case, it is obvious that the company not only has cooperative marketing, but also contains combined marketing strategy in the marketing process. It is worth of our reference. It is well-known that average profit of the enterprise will be declined with industry development. If an enterprise still depends on single product mode or homogeneity products for market competition, the enterprise cannot meet diversified and personalized consumption demand of consumers on one hand, it even can be separated from the market, which can be eliminated by the market on the other hand. Product updating speed is fast especially for IT enterprises. It is required that enterprises can plan the products on the basis of product competition strategy in the process of participating in marketing. Intel establishes stores around the world, and forms a complete set of marketing system, thereby ensuring product synchronization in the sales level so that market feedback information can be timely accepted, thereby improving the products.

Emphasis of Experience Marketing. Intel's booth gives people refreshing feeling for a long time. When Intel constantly introduces exhibitions with larger scale and higher quality, its first-class 
technology is shown in the exhibitions. It is worth mentioning that its success in CES is always an important foothold of Intel in market field development. Most users contacting with Intel can not forget the feeling thereof in 'Intel experience'. They seemingly touch the future. In addition, magic multimedia cascade display marks that Intel workers are always striving to diffuse own information faster than the market. The effect cannot be reached by two-dimensional advertising, television and radio. It is obvious that Intel makes people constantly improve their understanding on the products thereof by the aid of side experience. Current situation of Chinese electronic enterprise shows that many electronic products cannot be experienced by clients. Many consumers do not trust products without personal experience. Therefore, clients cannot purchase easily. We should admit that experience marketing actually can provide customers with comprehensive experience through sensory stimuli on customers, thereby showing core value of brand.

\section{Conclusion}

In summary, electronic product marketing field has many problems that should be perfected in China. Marketing experience of Intel Company can be adopted as reference for assisting us to observe the market direction more accurately. Electronic product enterprises in China should establish novel strategic development consciousness, realize own market positioning, and take effective measures to achieve breakthrough.

\section{References}

[1] He Jing. Research on culture marketing strategy case of LG Electronics (China) Company. Dalian University of Technology, 2014.

[2] Zhang Honghong. Research on HTC terminal marketing strategy. Nanjing University of Posts and Telecommunications, 2014.

[3] Yang Mu. Research on marketing case of Dalian Lianda Company. Dalian University of Technology, 2013.

[4] Sun Jing, Zhao Jing. Analysis on international market marketing strategy of electronic products. China Business \& Trade, 2011, 14:215-216.

[5] Xu Aiping. Analysis on experiential marketing strategy improvement path of consumption electronic products. China Business \& Trade, 2015, 08:3-5.

[6] Wang Xin. On marketing teaching method of electrical and electronic products. Scientific and Technical Information, 2013, 10:242;

[7] Chen Yitong. Enterprise management software market analysis and marketing strategy. Cuide to Business, 2015, 07: 69+63.

[8] Wang Xiaomin, Guo Jiakai, Zhang Beibei. 'Communication wall' pushed back by software marketing [J]. Software and Information Service, 2012, 05:28-39.

[9] Song Chengbing, Xu Ping. Exploration of efficient marketing organization pattern of software enterprises. Business Culture (II), 2011, 01:183.

[10] Zhang Ying, Jiang Xincheng. Research on software product experience marketing [J]. China Business \& Trade, 2012, 08: 42-43+47; 